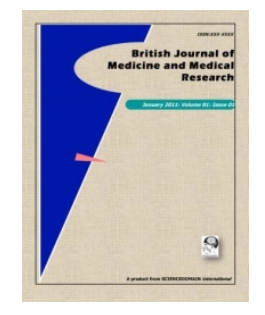

British Journal of Medicine \& Medical Research

3(2): 288-293, 2013

SCIENCEDOMAIN international

www.sciencedomain.org

\title{
Isolated Pancreas Fracture after a Go-Kart Accident
}

\author{
Engin Deniz Arslan ${ }^{1 *}$, Elif Çelikel ${ }^{1}$, Bige Sayın $^{2}$, Cemil Kavalcı $^{1}$, \\ Fevzi Yılmaz ${ }^{1}$, M. Serkan Yılmaz ${ }^{1}$ and Tamer Durdu ${ }^{1}$ \\ ${ }^{1}$ Department of Emergency Medicine, Ankara Numune Research and Training Hospital, \\ Ankara, Turkey. \\ ${ }^{2}$ Department of Radiology, Ankara Numune Research and Training Hospital, Ankara, \\ Turkey.
}

\section{Authors' Contributions}

Authors EDA, EC and BS designed and wrote the manuscript. Authors CK, FY and MSY evaluated and managed literature searches and author TD had some philosophic contributions to manuscript. All authors read and approved the final manuscript.

Case Study

Received $2^{\text {nd }}$ November 2012

Accepted $28^{\text {th }}$ December 2012

Published $19^{\text {th }}$ January 2013

\section{ABSTRACT}

Isolated pancreatic trauma is extremely rare because of pancreas' anatomic localization. Also, diagnosis of pancreatic injury may be difficult due to lack of sensitivity of initial clinical findings, laboratory and imaging examinations in emergency department. Morbidity and mortality is much higher in delayed presentation or if the trauma is unrecognized. In this paper we report a 20 -year-old female with isolated pancreas fracture after a blunt abdominal trauma due to a go-kart accident. Repeated evaluation of patient by taking into account of mechanism of trauma and suspicion of pancreatic injury is essential for early diagnosis.

Keywords: Pancreas fracture; trauma.

\section{INTRODUCTION}

Pancreas is located in the retroperitoneum in front of the vertebral column, in a relatively protected position. Abdominal muscles and adjacent abdominal organs provide anterior 
protection while the bony structure of thorax and dorsal muscle groups provide posterior protection of pancreas from blunt trauma. Therefore significant force is required to injure pancreas in a blunt trauma that explains paucity of isolated pancreatic injury.

Pancreatic injury account for less than $5 \%$ of patients with major abdominal injuries and majority of them result from penetrating trauma [1]. Blunt abdominal trauma may result in contusion, capsular tear or fracture of the pancreas, which might occur with or without ductal disruption. Evaluation of main pancreatic duct status is essential because ductal disruption is the main cause of pancreas specific morbidity. Parenchymal fracture is the most devastating result of pancreatic injury and occurs due to the displacement of the pancreas against the vertebral column in a blunt trauma. Motor vehicle accidents are the most common injury mechanism in blunt pancreatic trauma. The most common organs injured with the pancreas are spleen and liver [2]. In practice, pancreatic injuries are usually diagnosed during emergency laparotomy that is performed to treat accompanying intraperitoneal visceral or vascular injuries.

\section{CASE}

A 20-year-old female was admitted with a history of blunt abdominal trauma after a go-kart racing accident. She denoted that she collided to barriers and hit her upper region of abdomen to the steering wheel and being ejected from the go-kart. On admission, the patient reported pain in the upper abdomen, with normal vital signs and physical examination except mild tenderness in the epigastrium. Her initial ultrasonographic examination and laboratory findings were also in normal limits. During observation her pain progressed and her second ultrasonographic examination revealed free fluid accumulation at perisplenic, perihepatic, peripancreatic and pelvic area. The amylase level increased to $1321 \mathrm{IU} / \mathrm{L}$ (normal limits: 36-128 IU/L) with white blood cell count (WBC) $14600 / \mu \mathrm{L}$, five hours after trauma. The patient' contrast enhancement abdominal computed tomography was obtained and showed an isolated pancreatic fracture (Fig. $1 \& 2$ ). The patient had a grade III injury according to American Association of the Surgery of Trauma (AAST) classification of pancreatic injury. The patient was taken to the operating room and distal pancreatectomy with spleen preservation was performed. The postoperative period was uneventful and the patient was discharged on the $10^{\text {th }}$ day of admission.

\section{DISCUSSION}

Pancreatic injuries are very rare and account for less than $5 \%$ of severe abdominal injuries [1]. In a retrospective review over a ten year period, it has been reported that pancreas was involved in $1.1 \%$ of patients with penetrating injuries compared to $0.2 \%$ with blunt injuries [3]. Overall, up to $25 \%$ of pancreatic injuries arise from blunt trauma whereas pancreatic injury is observed in $1-5 \%$ in blunt abdominal trauma. [4] Additionally Timberlake demonstrated an average of 2.5 associated organ injuries at operation in patients with pancreatic trauma indicates that isolated pancreatic trauma is very rare [5]. 


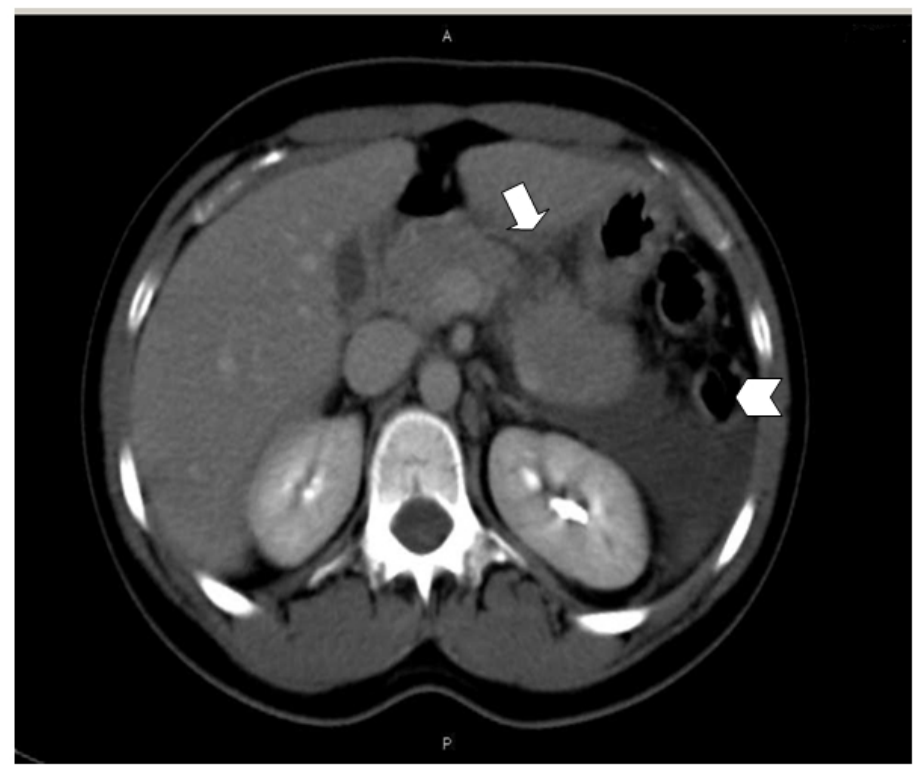

Fig. 1. Contrast enhancement $\mathrm{CT}$ revealed pancreatic fracture resulting from go-kart accident

White arrow: Pancreatic disruption of the pancreas body White notched arrow head: Peripancreatic-perirenal free fluid accumulation

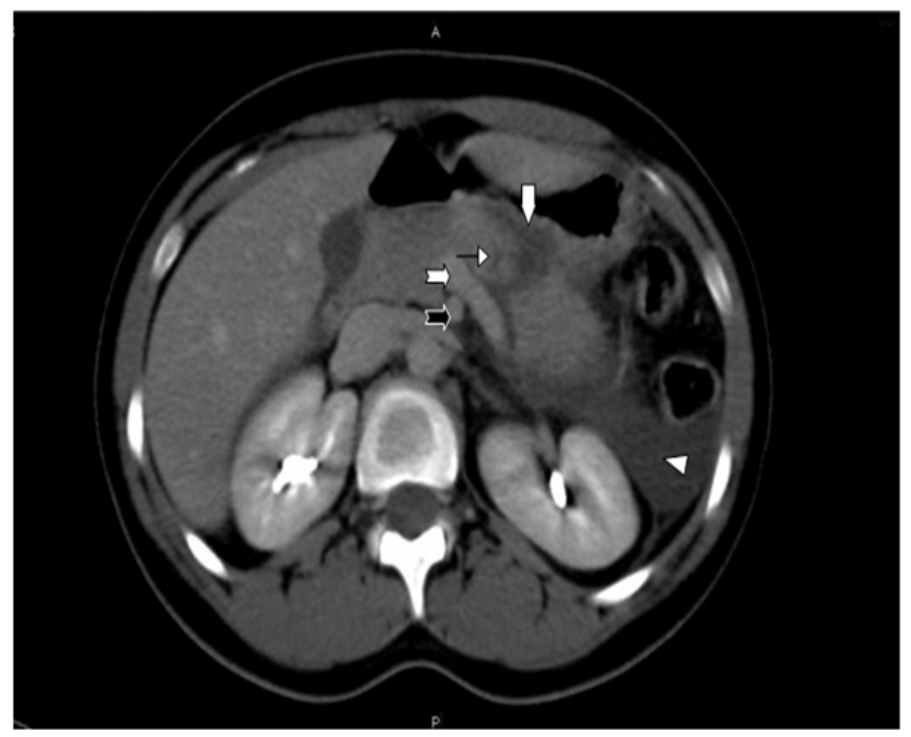

Fig. 2. Pancreas fracture with main pancreatic duct injury. Note that proximity of pancreas to the vascular structures.

White arrow: Fracture of the pancreas body

Thin arrow: Laceration of the pancreatic duct.

White arrow head: Peripancreatic-perirenal free fluid accumulation

Notched white arrow: Splenic vein

Notched black arrow: Superior mesenteric artery 
In isolated pancreatic injury, initial physical signs and laboratory findings are often nonspecific. Diffuse or epigastric abdominal pain, tenderness and abdominal wall ecchymoses may be present. The serum amylase level on admission is of little value in the diagnosis of acute injury to the pancreas. The elapsed time from injury to admission seems to be the major factor that influences the serum amylase level and the serum amylase level is not diagnostic within 3 hours of trauma [6]. In our case, initial physical and laboratory findings were not remarkable and progression was observed within few hours of the event, which was compatible with the literature.

As computed tomography (CT) is commonly employed in stable blunt trauma patients we confirmed pancreas fracture with CT imaging. CT appearance of pancreatic injury ranges from a normal initial appearance of the pancreas to active pancreatic bleeding. Holmes et al reported that after normal abdominal CT scans, $0.3 \%$ patients had intraabdominal injury mostly attributed to pancreatic injury [7]. Actually pancreatic parenchymal injury can be readily demonstrated with CT imaging but magnetic resonance (MR) cholangiopancreatography is indicated for direct imaging of the pancreatic duct and sites of disruption [8].

Patients with pancreatic injury may be managed with operation or non-operative observation according to the pancreatic duct status and location of the injury in relation to superior mesenteric vessels. Patients with documented intact ductal systems (AAST Grade I-II) may be drained and observed. Patients with documented ductal injury require distal pancreatectomy for AAST Grade III, pancreaticoojejunostomi for AAST Grade IV or pancreaticoduedonectomy for AAST Grade V [4]. In our case ductal disruption was suggested based on the extent of the parenchymal laceration and the patient was taken to immediate laparatomy and distal pancreatectomy was performed. Govaert et al reported two cases of pancreas fracture due to go-kart accident similar to ours in whom pancreaticojejunostomi were performed [9]. The difference between type of surgeries probably depend on the difference between grading of injuries.

Pancreatic fistula, pancreatic abscess, traumatic pancreatitis, and pseudocyst formations are the major complications of the pancreatic injury. Delay in diagnosis and surgery show higher rate of pancreas-specific morbidity reaching up to $60 \%$ [10]. Olah et al reported that all patients requiring delayed surgical intervention had pancreas-specific morbidity [11].

Krige et al. reported the mortality rate of $16 \%$ in a study over 17 years involving 110 patients with blunt pancreatic trauma [12]. Mortality in patients with pancreatic trauma in the early period is due to vascular and other associated injuries and primarily not related to the pancreatic injury. The mortality increases with higher grades of the pancreatic injuries, presence of shock on admission, and the number of associated injuries.

In English literature there are also some other reports of isolated pancreatic injury due to blunt abdominal trauma. The mechanism of these injuries were as follows; motorcycle accident [13], car accident [14], trauma during soccer game [15], fall from height with hitting to the handle of a ladder [16] and two with go-kart accident [9]. All patients had severe blunt trauma characterized by direct blow to the central abdomen, normal vital findings, initial subtle physical findings that progressed within hours, elevated amylase levels and no associated organ injury. In these injuries; traumatic force might be not enough to injure the adjacent organs but it might be more focused to move the pancreas against the vertebral column resulting in pancreatic fracture. Although the grade of the pancreatic injuries in our 
patient and in other patients who had pancreas fractures was high, the patients survived most probably owing to the absence of associated injuries and early surgical treatment.

\section{CONCLUSION}

We may encounter patients with pancreatic injury which shows subtle clinical findings, and the pancreatic injury cannot be detected through initial laboratory and imaging investigations. Repeated evaluation of the patient taking into account the mechanism of trauma and suspicion of pancreatic injury is essential for early diagnosis and the survey should be excellent with early diagnosis and proper management. Also, history of go-kart accident might alert physician for pancreatic injury even in the absence of other traumatic lesions.

\section{COMPETING INTERESTS}

Authors have declared that no competing interests exist.

\section{REFERENCES}

1. Beckingham IJ, Krige JE. ABC of diseases of liver, pancreas, and biliary system: Liver and pancreatic trauma. BMJ. 2001;322:783-5

2. Bradley EL 3rd, Young PR Jr, Chang MC, Allen JE, Baker CC, Meredith W, et al. Diagnosis and initial management of blunt pancreatic trauma. Guidelines From a Multiinstitutional Review Annals Of Surgery. 1998;227:861-869

3. Akhrass R, Yaffe MB, Brandt CP, Reigle M, Fallon WF Jr, Malangoni MA. Pancreatic trauma: a ten-year multi- institutional experience. The American Surgeon. 1997;63:598-604.

4. $\quad$ Ahmed N, Vernick JJ. Pancreatic injury. South Med J. 2009;102:1253-6.

5. Timberlake GA. Blunt pancreatic trauma: experience at a rural referral center. Am Surg. 1997;63:282-6.

6. Takishima T, Sugimoto K, Hirata M, Asari Y, Ohwada T, Kakita A. Serum amylase level on admission in the diagnosis of blunt injury to the pancreas: its significance and limitations. Ann Surg. 1997;226:70-6.

7. Holmes JF, McGahan JP, Wisner DH. Rate of intra-abdominal injury after a normal abdominal computed tomographic scan in adults with blunt trauma. Am J Emerg Med. 2012; 30,574-9.

8. Gupta A, Stuhlfaut JW, Fleming KW, Lucey BC, Soto JA. Blunt trauma of the pancreas and biliary tract: a multimodality imaging approach to diagnosis. Radiographics. 2004;24:1381-95.

9. Govaert MJ, Ponsen KJ, de Jonge L, de Wit LT, Obertop H. Fracture of the pancreas in two patients go-kart accident. HPB. 2001;3:3-6.

10. Lin BC, Chen RJ, Fang JF, Hsu YP, Kao YC, Kao JL. Management of blunt major pancreatic injury. J Trauma. 2004;56:774-8.

11. Oláh A, Issekutz A, Haulik L, Makay R. Pancreatic transection from blunt abdominal trauma: early versus delayed diagnosis and surgical management. Dig Surg. 2003;20:408-14.

12. Krige JE, Kotze UK, Hameed M, Nicol AJ, Navsaria PH. Pancreatic injuries after blunt abdominal trauma: an analysis of 110 patients treated at a level 1 trauma centre. S Afr J Surg. 2011;49:62-4. 
13. Fonseca AZ, Ribeiro MAF, Contrucci O, Pompeo A, Orsetti A, Neto HA. Spleen preserving distal pancreatectomy in an isolated blunt pancreatic trauma. World $\mathrm{J}$ Gastrointest Surg. 2011;27:138-141.

14. Brestas PS, Karakyklas D, Gardelis J, Tsouroulas M, Drossos C. Sequential CT evaluation of isolated non-penetrating pancreatic trauma. JOP. 2006;11:51-5.

15. Dobrinja C, Roseano M, Pravato M, Liguori G. Pancreatic Injury Following Blunt Abdominal Trauma during a Soccer Game. Phys Sportsmed. 2008;36:115-8.

16. Viti M, Papis D, Ferraris V, Fiori F, D'Urbano C. Isolated and complete traumatic rupture of the pancreas: A case report and a review of the literature. Int J Surg Case Rep. 2012;3:590-3.

(c) 2013 Arslan et al.; This is an Open Access article distributed under the terms of the Creative Commons Attribution License (http://creativecommons.org/licenses/by/3.0), which permits unrestricted use, distribution, and reproduction in any medium, provided the original work is properly cited.

Peer-review history:

The peer review history for this paper can be accessed here:

http://www.sciencedomain.org/review-history.php?iid=177\&id=12\&aid=846 